\title{
Measurement of concentration dependency of diffusion coefficient in ethanol-water solution under different storage condition
}

\author{
Atsuki KOMIYA*, Kengo SATO** and Junnosuke OKAJIMA* \\ * Institute of Fluid Science, Tohoku University \\ 2-1-1, Katahira, Aoba-ku, Sendai, 980-8577, Japan \\ E-mail: komiya@tohoku.ac.jp \\ ** Graduate School of Engineering., Tohoku University \\ 6-6, Aoba, Aramaki-aza, Aoba-ku, Sendai, 980-8579, Japan
}

Received: 4 April 2018; Revised: 23 July 2018; Accepted: 7 August 2018

\begin{abstract}
This study focuses on the diffusion phenomena in ethanol-water systems. The diffusion coefficient of ethanol-water systems has a strong concentration dependency originating from intermolecular interactions and the association of molecules in solution. Recently, it has been suggested that flavor variation in whiskey is partly due to different storage conditions that can affect molecular structure variation. To evaluate the molecular structure variation, diffusion coefficient measurements are considered effective. To quantitatively evaluate the variations in the diffusion process and measure the mass diffusion coefficient, the analysis method was improved to measure the concentration field more precisely. Two ethanolic solutions were prepared in this study: one preserved under normal conditions and the other was stored under isothermal conditions for 496 days. By comparing diffusion processes occurring under different storage conditions, the different diffusion coefficients were determined and the effect of storage conditions was discussed. The diffusion coefficient of the ethanolic solution stored under an isothermal condition was smaller than that of the solution stored under normal conditions.
\end{abstract}

Keywords : Diffusion coefficient, Ethanol, Phase-shifting interferometer, Concentration dependency

\section{Introduction}

A better understanding of mass transport phenomena in the liquid phase is essential for the fields of biochemical and food engineering. The diffusion coefficient is difficult to measure precisely due to the complexity of analytical procedures and difficulty in controlling measurement precision. In addition, the solution concentration must be considered, because the diffusion coefficient of the solution is often concentration dependent, but is technically difficult to measure precisely. It is presumed that the concentration dependency is caused by intermolecular interactions and the association of molecules in solution (Harris et.al. 1993, 1998).

Flavor variation of whiskey preserved under isothermal conditions has been recently reported. Pseudo molecular weight change under isothermal conditions may explain this variation (SUNTORY News release 2015). Considering this supposition, the mass diffusion process of alcohol might also vary under these storage conditions.

To date, the diffusion phenomena of ethanol-water systems have been extensively studied experimentally and numerically, and diffusion coefficients were determined (Pratt et al. 1974; Königer et al. 2009; Q. Zhu et al. 2016; Moggridge, 2012). In these studies, it was assumed that the mass diffusion coefficient of ethanol in water was constant for evaluating the diffusion field. Therefore, for systems with a concentration dependence of mass diffusion coefficients, the measurement of small concentration differences, (where the mass diffusion coefficient was treated as constant), has been performed periodically and the concentration dependency was evaluated using the accumulated data. On the contrary, the proposed method in this study treats the diffusion coefficient as a function of concentration, and the diffusion 
coefficient and its dependency over a wide range of concentration can be measured at once. The method in detail is described in sub-section 2.3.

Whiskey is a liquor with $40 \%$ ethanol concentration. When evaluating the concentration dependence of the diffusion coefficient in a $40 \%$ alcohol solution, a dilution process is required to form an analyzable concentration field. However, this dilution changes the intermolecular interactions and association of molecules, i.e. pseudo molecular weight in solution. Thus, the $40 \%$ alcohol solution must be used without dilution to evaluate different preserving conditions. When measurements are performed without dilution, the influence of intermolecular interactions and associations on the diffusion process is more pronounced. Therefore, transient diffusion field formation with high concentration difference between the $40 \%$ alcohol solution and pure water is required.

The objective of this study was to experimentally evaluate the variation of diffusion coefficients due to different storage conditions. First, the conventional measurement technique was improved such that the measurement of concentration fields of high concentration differences, such as a $40.0 \mathrm{wt} \%$ ethanol solution, could be performed. The diffusion coefficient was measured using this improved phase-shifting interferometer. A $40.0 \mathrm{wt} \%$ ethanol aqueous solution, which is the main component of whiskey, was used in this study. The diffusion process in various ethanol aqueous solutions was compared and the influence of preserving conditions on diffusion phenomena was experimentally evaluated.

\section{Experimental set-up and procedure}

\subsection{Experimental set-up}

The transient diffusion field of a binary liquid system between $40.0 \mathrm{wt} \%$ ethanol aqueous solution and purified water was visualized using phase-shifting interferometer (Komiya et al. 2006; Torres et al., 2012). A schematic of the measurement system is shown in Fig. 1. For precise measurement of the transient diffusion field of a large concentration difference, a phase-shifting technique was used in the visualization system. The emitted laser intensity exhibited a Gaussian distribution, as shown in Fig. 1, and only the core region of the distribution was used to maintain the intensity for basic illumination for visualization. Three images were required for the technique, and $I_{1}, I_{2}$, and $I_{3}$ were obtained by rotating a polarizer settled in front of the detector. The experiments were performed using the same as the method proposed by Torres et al. (Torres et al. 2012).

A test cell cuvette made of quartz glass was used to keep the optical path length constant against thermal expansion due to laser irradiation. The size of test cell was $0.5 \mathrm{~mm}$ in optical path length, $45 \mathrm{~mm}$ in height, and $10 \mathrm{~mm}$ in width. To avoid natural convection due to buoyancy, the higher density solution (purified water) was first injected at the bottom of the test cell cuvette. Subsequently, the low concentration solution was injected smoothly and calmly to minimize initial disturbances. During the visualization of the transient diffusion field, the phase-shifted data was measured every 10 min after $60 \mathrm{~min}$ passed from the diffusion field formation to prevent measuring the initial disturbance effect.

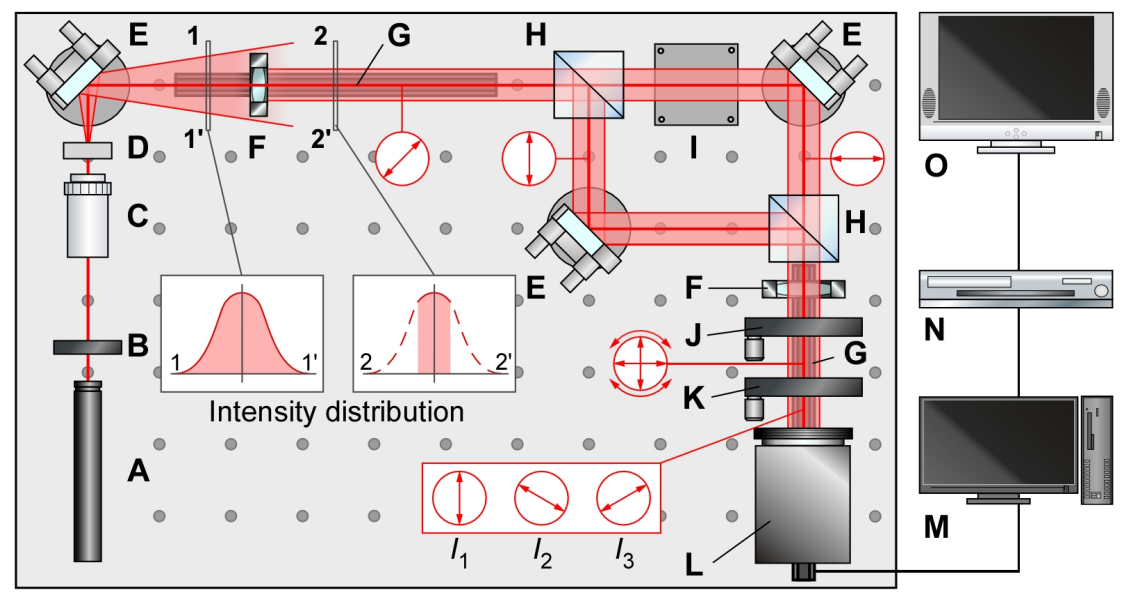

A He-Ne-laser
B polarizer
C objective-lens
D pin-hole
E mirror
F convex lens
G optical rail
H polarized beam splitter
I cell stage
J quarter-wave plate
K rotating polarizer
L detector
$\mathbf{M}$ image processor
$\mathbf{N}$ recorder
$\mathbf{O}$ monitor

Fig. 1 Schematic of the measurement system used for the transient diffusion field. The basic arrangement of interferometer is Mach-Zehnder type. A phase-shifting technique was used for precise visualization of the transient diffusion field of a large concentration difference. 


\subsection{Concentration dependence of the refractive index}

For ethanol aqueous solutions, the concentration dependence of the refractive index over a wide concentration range is not linear, as shown in Fig. 2. Especially, in the high concentration region $(70-80 \mathrm{wt} \%)$, reduced concentration dependency is observed, and a negative relation was found in the range of $80-100 \mathrm{wt} \%$. The black symbols represent reference data (Herráez et al. 2006), and the red symbols indicate experimental data obtained using a phase-shifting interferometer in a previous study, and the blue line is the linear approximation of the experimental data up to $40 \mathrm{wt} \%$ (Sato et al. 2017). To achieve accurate concentration distribution data over a wide range of concentration, the nonlinearity of concentration dependence should be considered. However, for ethanol aqueous solutions up to $40 \mathrm{wt} \%$, the dependency can be assumed to be linear, as shown in Fig. 2. The largest difference between linear and quadratic approximations within this concentration range is $|n|=0.0136$ at a concentration of $40.0 \mathrm{wt} \%$. This causes less than $1.0 \%$ difference of refractive index and could be negligible. Therefore, the concentration distribution was obtained directly from the difference in refractive index obtained by phase-shifted data.

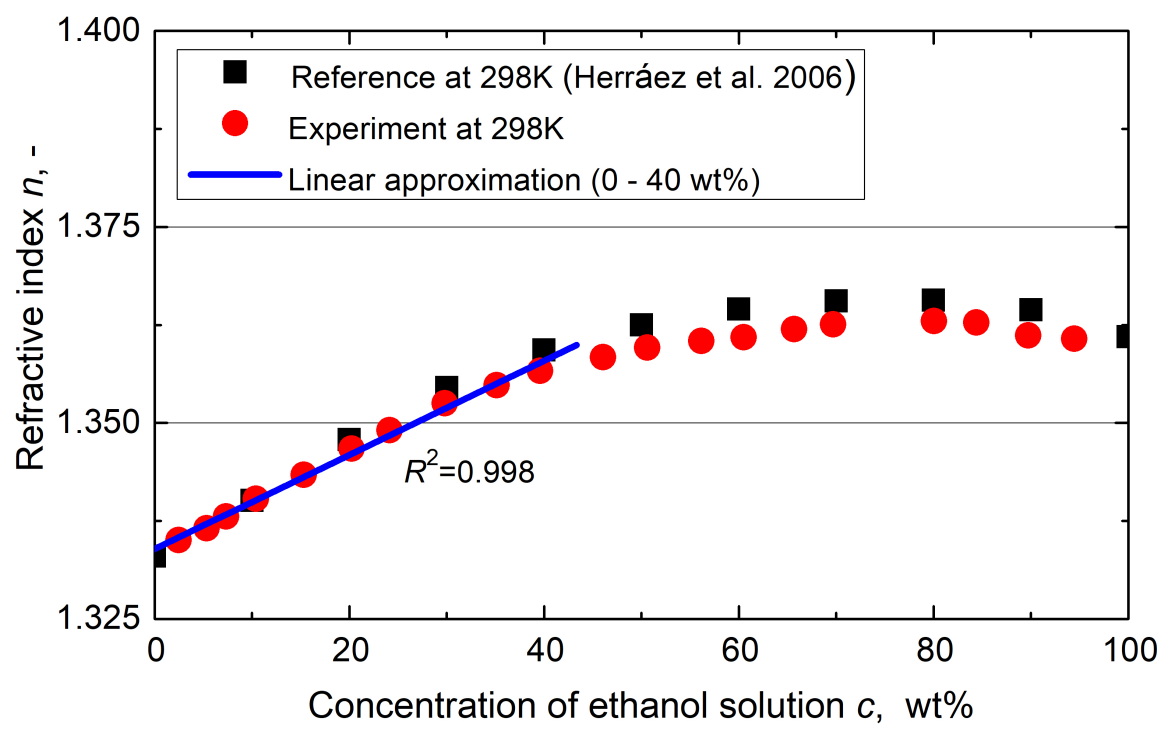

Fig. 2 Relationship between concentration of ethanol aqueous solution and refractive index with the reference values from Herráez and Belda (2006). Within the concentration range of 0 to $40.0 \mathrm{wt} \%$ ethanol, the relation is assumed to be linear. Over the entire concentration range, the relation changes to be non-linear.

The process used to acquire the concentration distribution from the phase-shifted data is shown in Fig. 3. Figure 3(a) shows a typical phase-shifted image of the diffusion field, and Fig. 3(b) shows the phase difference distribution obtained from the difference of refractive index, from 0 to $\pi$ and intensity data from 0 to 255, along the dashed line A to B in Fig. 3(a). Figure 3(c) shows the absolute phase difference obtained by connecting the phase-shifted data at discontinuous points. From the phase difference distribution, the spatially precise concentration distribution was obtained as shown in Fig. 3(d).

\subsection{Inverse analysis to determine diffusion coefficients and their concentration dependence}

The mass diffusion coefficient of ethanol in aqueous solution and its concentration dependence was determined using the inverse analysis method comparing the experimental and simulation data. In this study, diffusion process was assumed to be isothermal and one-dimensional free diffusion. The governing equation of this diffusion process can be approximated as

$$
\begin{aligned}
& \frac{\partial C}{\partial t}=\frac{\partial}{\partial x}\left(D(C) \frac{\partial C}{\partial x}\right), \\
& D(C)=D_{0}+\alpha C+\beta C^{2},
\end{aligned}
$$




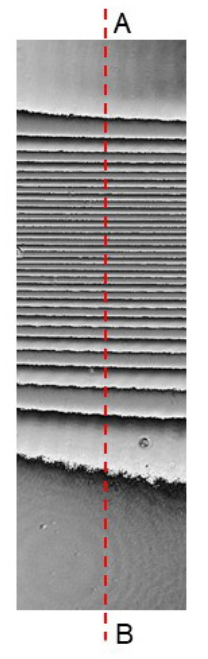

(a)

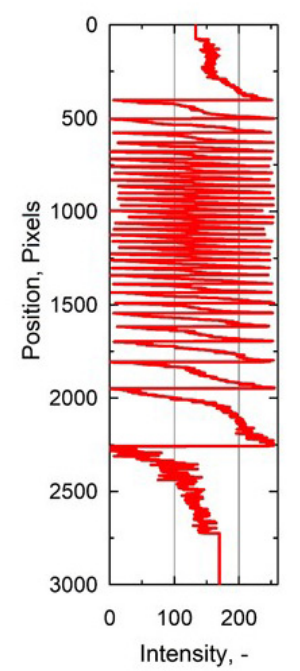

(b)

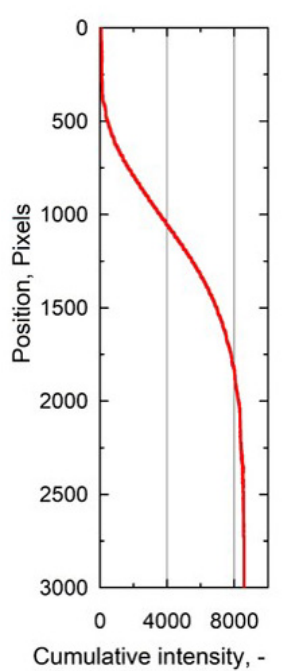

(c)

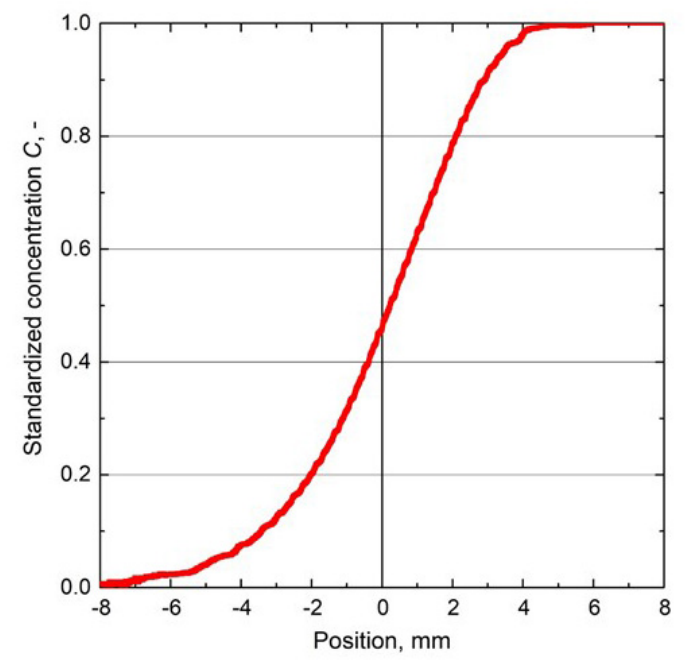

(d)

Fig. 3 Acquisition of the concentration distribution from phase-shifted data. (a) Typical diffusion field of binary system visualized by phase-shifted data. (b) The 0 - 255 intensity distribution along the dashed line A - B. (c) Unwrapped phase-shifted data as a cumulative intensity. (d) Concentration distribution obtained from the phase-shifted data.

where $x[\mathrm{~m}]$ is location, $t[\mathrm{sec}]$ is time, $C[-]$ is the standardized concentration, and $D\left[\mathrm{~m}^{2} / \mathrm{s}\right]$ is the mass diffusion coefficient which is a function of concentration. Using $D_{0}, \alpha$, and $\beta$ as coefficients which represent concentration dependence of the mass diffusion coefficients, the value of the mass diffusion coefficient and its concentration dependence could be determined. In the numerical simulation, Eq. (1) was discretized using the finite volume method and solved by complete implicit solution method using a tentative mass diffusion coefficient, $D_{\text {cal }}\left[\mathrm{m}^{2} / \mathrm{s}\right]$, which is defined by Eq. (2). The boundary conditions are $C=1$ at $x \rightarrow \infty$ and $C=0$ at $x \rightarrow-\infty$. For the initial conditions, a concentration distribution was obtained from the visualization experiment at the early stages of diffusion and used. At an arbitrary elapsed diffusion time, the difference between the experimental and numerical concentration distribution was compared using an objective function $\varphi[\mathrm{m}]$ for inverse analysis, which is given by

$$
\varphi\left(D_{\text {cal }}\right)=\int_{-\infty}^{\infty}\left|C_{\exp }(x)-C_{\text {cal }}\left(x, D_{\text {cal }}\right)\right| d x,
$$

where $C_{\text {exp }}(x)$ is the experimental concentration distribution, and $C_{\text {cal }}\left(x, D_{\text {cal }}\right)$ is that from the numerical simulation. As described in Section 2.2, the high resolution spatial data was obtained from the experimental study, therefore both concentration values at each spatial point could be compared so that the discretized number in the simulation was adjusted to the pixel number of the detector shown in Fig. 1. To determine the diffusion coefficient as a function of concentration, a sort of curve fitting method was applied. The experimental and numerical results give the concentration values at the exactly same position of diffusion field because Eq. (2) was discretized with taking into account of pixel number of the experimental field of view. To minimize the integrated difference value of concentration between experimental and numerical results in the target concentration range, $\varphi\left(D_{c a l}\right)$, which is expressed in Eq. (3), the diffusion coefficient and its dependency are determined. The objective function $\varphi\left(D_{c a l}\right)$ was minimized using the non-linear conjugate gradient method (Okajima et al. 2008).

\section{Accuracy of the proposed method for diffusion coefficient determination}

The transient diffusion field of the binary system between $40.0 \mathrm{wt} \%$ ethanol aqueous solution and purified water was visualized by phase-shifted data shown in Fig. 4, which were obtained from a high-resolution CMOS sensor (7952 in vertical $\times 4472$ in horizontal pixels). For the experimental conditions in this study, the spatial resolution of the measurement system was $5.5 \mu \mathrm{m}$ per pixel and $0.005 \mathrm{wt} \%$ per intensity.

As described above, the concentration distribution could be obtained directly from the phase difference data. Figure 5 shows concentration distributions obtained from Fig. 4. 

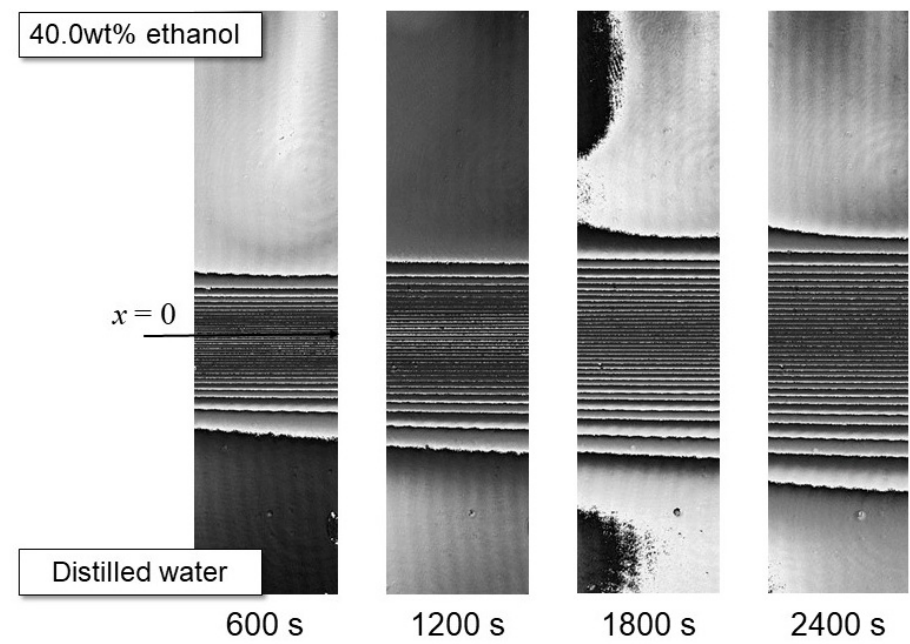

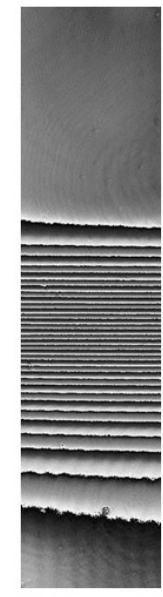

$3000 \mathrm{~s}$

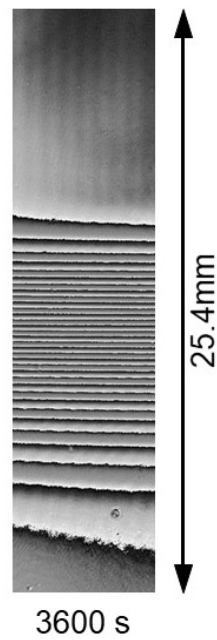

$3600 \mathrm{~s}$

Fig. 4 Transient diffusion field visualized by phase-shifted data. After $10 \mathrm{~min}$ from the formation of the diffusion field, the image reveals that the diffusion field could be assumed to be a one-dimensional process. The concentration field extended as the time advanced.

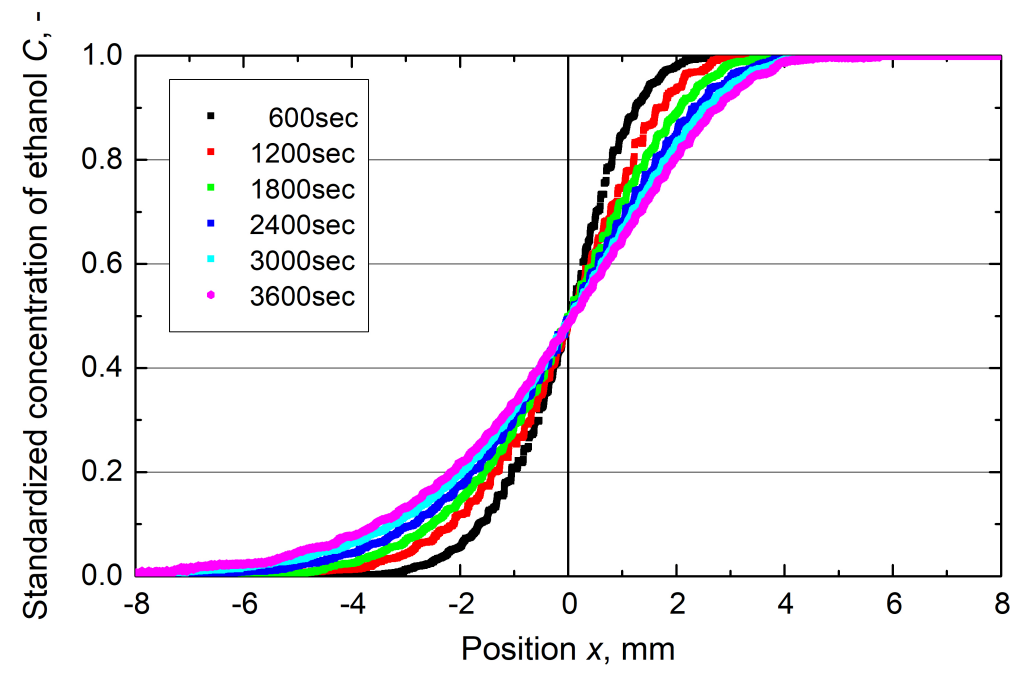

Fig. 5 Concentration distributions of the transient diffusion field obtained from the phase difference data. Through the diffusion process, spatially high-resolution data could be obtained. It is clear that the diffusion fields show an asymmetrical distribution with respect to the origin.

The diffusion coefficients were determined by minimizing the objective function defined in Eq. (3). The determined diffusion coefficients and concentration dependencies at each measurement time are listed in Table 1, and vary irregularly. Comparing the results between $1200 \mathrm{~s}$ and $3600 \mathrm{~s}$, both results represent close values of literature data. However, the determined values temporally scattered, and reached to certain values. It is likely that the temporal variations for $1200 \mathrm{~s}$ to $2400 \mathrm{~s}$ are due to remaining initial disturbance, measurement errors in the concentration distribution (Fig. 5), and analytical errors. This indicates that the variation was mainly caused by the remaining initial disturbance and appropriate values could be obtained $3600 \mathrm{~s}$ after the formation of the diffusion field for the conditions used in this study.

Figure 6 shows the relationship between ethanol concentration in aqueous solution and the diffusion coefficient determined by inverse analyses, in which the experimental data stored under normal condition were applied. Here, the definition of "normal conditions" refer to temperature distribution and variation in time, typically found in storage facilities in normal brewery without temperature control. Seasonally the surrounding temperature varies within approximately $\pm 3 \mathrm{~K}$ without any temperature control. The solution was stored for over 490 days and it is not controlled in temperature precisely. For comparison, reference data for the diffusion coefficients of ethanol-water solutions (Pratt et al. 1974; Königer et al. 2009; Hao et al. 1996) and empirical relations are shown in Fig. 6. The vertical bars correspond to the standard deviations of the experiments performed in triplicate under the same conditions. Table 2 lists the diffusion coefficients determined from the reference and present data at each concentration shown in Fig. 6. In additions, it shows 
the difference between the reference and present data. The determined diffusion coefficients and their dependencies agree well with the reference data within a maximum difference of $8.22 \%$. This indicates that the measurement proposed in this study is accurate for the evaluation of diffusion coefficients and their concentration dependencies.

Table. 1 Determined coefficients at each measurement time acquired by inverse analyses.

\begin{tabular}{|c|c|c|c|c|c|c|c|}
\hline & Literature data* & $1200 \mathrm{~s}$ & $1800 \mathrm{~s}$ & $2400 \mathrm{~s}$ & $3000 \mathrm{~s}$ & $3600 \mathrm{~s}$ & Average \\
\hline$D_{0} \times 10^{-9}\left[\mathrm{~m}^{2} / \mathrm{s}\right]$ & 1.24 & 1.2182 & 0.9652 & 1.0555 & 1.1950 & 1.2562 & 1.1380 \\
\hline$\alpha \times 10^{-9} \quad\left[\mathrm{~m}^{2} / \mathrm{s}\right]$ & -1.19 & -1.1482 & -0.4426 & -0.5461 & -1.0236 & -1.2613 & -0.8844 \\
\hline$\beta \times 10^{-9} \quad\left[\mathrm{~m}^{2} / \mathrm{s}\right]$ & 0.32 & 0.3553 & -0.0459 & -0.0449 & 0.3249 & 0.5252 & 0.2229 \\
\hline
\end{tabular}

*determined by quadratic fitting of reference data (Pratt et al. 1974; Königer et al. 2009; Hao et al. 1996)

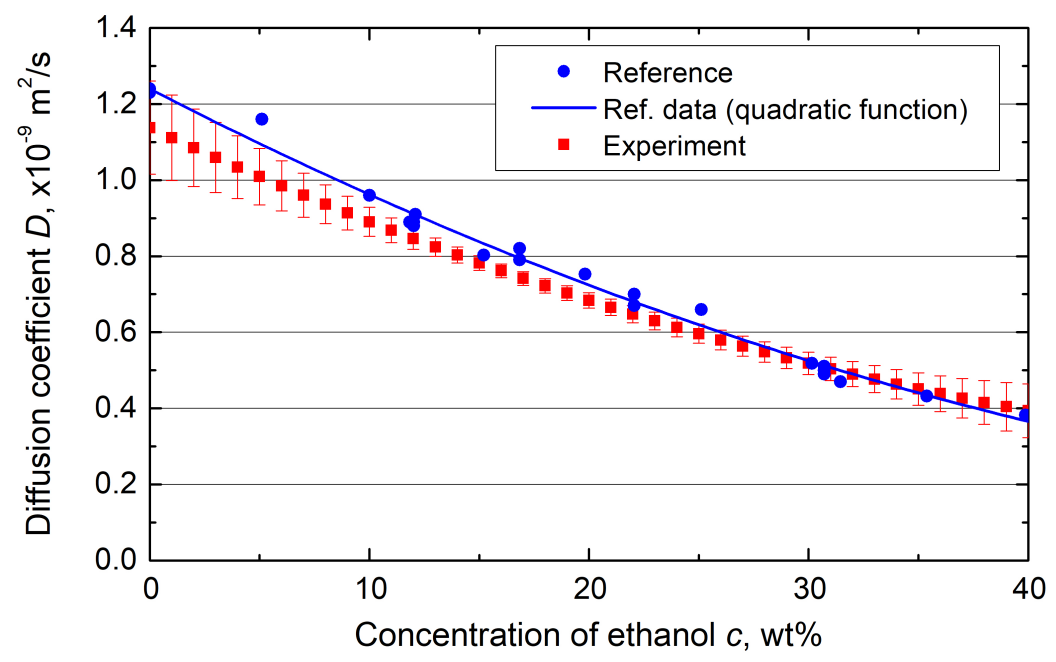

Fig. 6 Relationship between the concentration of ethanol in aqueous solution and diffusion coefficients. The present data show good agreement with the reference data and their empirical relations over a wide range of ethanol concentrations.

Table. 2 Concentration dependence of the diffusion coefficients of ethanol-water systems as calculated by inverse problem analyses.

\begin{tabular}{c|ccc}
\hline \hline ethanol concentration, wt $\%$ & $D_{\text {ref., }} \times 10^{-9}\left[\mathrm{~m}^{2} / \mathrm{s}\right]$ & $D \times 10^{-9}\left[\mathrm{~m}^{2} / \mathrm{s}\right]$ & Relative difference, $\%$ \\
\hline 0 & 1.24 & 1.14 & 8.22 \\
10 & 0.96 & 0.89 & 7.46 \\
20 & 0.72 & 0.68 & 5.54 \\
30 & 0.53 & 0.52 & 1.33 \\
40 & 0.37 & 0.39 & 7.54
\end{tabular}

\section{Effect of storage condition on diffusion phenomena}

\subsection{Experimental samples}

As described in the introduction, it is thought that the structure of the molecules in solution change as a result of different storage conditions such as controlled/non-controlled thermal conditions (SUNTORY News release 2015). In this study, $40.0 \mathrm{wt} \%$ ethanol aqueous solution, which is the main component of whiskey, was stored for 496 days in an isothermal storage container. The container was composed of mainly thermal insulation material, but metal plates were also used. The multilayered structure of the insulation material and plate could spatially and temporally maintain the temperature within $\pm 40 \mathrm{mK}$. The diffusion process of ethanol stored in the container was compared to that stored under normal conditions. 


\subsection{Results and discussion}

The determined mass diffusion coefficients and concentration dependencies of the ethanol-water system are shown in Fig. 7. The black dots represent the diffusion coefficients of the ethanol solution stored under normal conditions and the red dots represent data obtained under isothermal conditions stored for 496 days. The vertical bars on the dots correspond to the standard deviations of the triplicate experiments performed under identical conditions. In both cases, the diffusion coefficients decrease with increasing ethanol concentration. Under normal conditions, the decreasing rate of diffusion coefficients as a function of concentration slightly changes, but the diffusion coefficient monotonously decreases under isothermal conditions within the concentration range of 0 to $40.0 \mathrm{wt} \%$. Comparing the results, the diffusion coefficient of the aqueous solution stored under isothermal conditions shows much smaller value than under normal conditions. This difference is remarkable in the dilute ethanol concentration range. From the viewpoint of molecular motion in solution, it is considered that the difference can be attributed to the variation of quasi-molecular structure in solution. One of the possibilities to explain this difference is that the ethanol molecules in dilute solutions might weakly cohere with surrounding water molecules and their pseudo molecular weight increases. On the other hand, in the high concentration range, less opportunity might be provided for the ethanol molecules to cohere with other molecules because the surrounding water molecules are more scare. Furthermore, the isothermal condition could partially assist in the coherence of ethanol molecules because the temperature in the system was spatially and temporally maintained and the internal energy in solution never changes. This slows the molecular motion compared with normal conditions.

From Fig. 7, it should also be noted that the diffusion coefficients under both isothermal/normal conditions within the concentration range from 30 to $40 \mathrm{wt} \%$ show identical values. It is well known that the diffusion coefficient of the ethanol-water system is minimized near the ethanol concentration of $40 \mathrm{wt} \%$ (Pratt et al. 1974; Königer et al. 2009; Hao et al. 1996). In this concentration range, the interaction between ethanol and water molecules, namely hydration, might cause increased kinematic viscosity, which minimizes the diffusion coefficient. The experimental results and their comparison show that the interaction is unaffected by the storage condition at the ethanol concentration of $40.0 \mathrm{wt} \%$, which is the main ingredients of normal whiskey. However, there is a fact that flavor variation in whiskey is due to different storage condition. There is a possibility that a flavor variation is caused by not only main ingredient of whiskey but also the other ones. A normal whiskey contains many ingredients. In future studies, these above hypotheses and relationship between flavor variation and change of diffusion phenomenon should be clarified and experimentally tested using not only ethanol-water system but also ethanol-water-additive system.

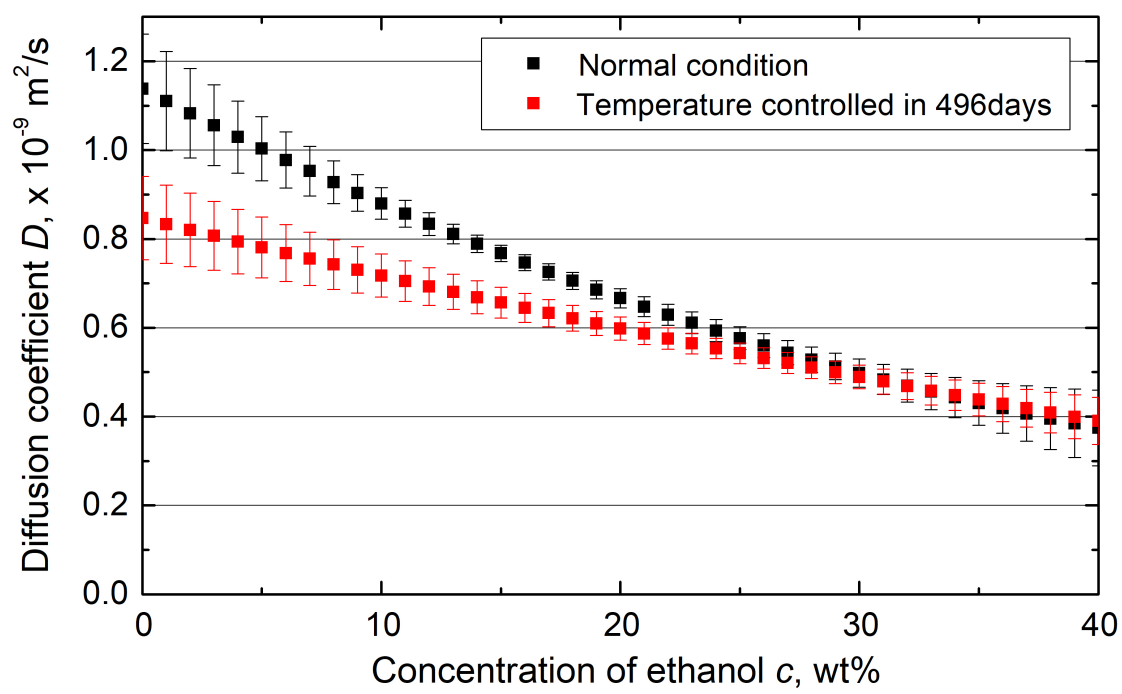

Fig. 7 Concentration dependencies of the mass diffusion coefficient in the ethanol-water system. Comparing the results, the storage condition strongly affects diffusion phenomenon and the dilute ethanol concentration range is especially affected. 


\section{Concluding Remarks}

In this study, the effect of storage condition on diffusion process in the ethanol-water system was investigated. The transient diffusion fields of the binary liquid system of $40.0 \mathrm{we} \%$ ethanol aqueous solution and purified water under two storage conditions were clearly visualized using a phase-shifting interferometer with a high spatial resolution. Despite the high concentration difference, the concentration dependency of diffusion coefficients was evaluated. The ethanol solution stored under isothermal condition exhibited much smaller mass diffusion coefficients than that stored under normal conditions. This experimental results could suggest that the ethanol molecules in dilute solutions might cohere weakly with surrounding water molecules and their pseudo molecular weight increases. For a more complete understanding of experimental results, additional parametric experiments are required in future work.

\section{References}

Chemistry Handbook, The Chemical Society of Japan, Maruzen (1993).

Hao, L., and Leaist, D.G., Binary mutual diffusion coefficients of aqueous alcohols. Methanol to 1-heptanol, Journal of Chemical \& Engineering Data, vol. 41, No. 2, (1996), pp. 210-213.

Harris, K.R., Goscinska, T., and Lam, H.N., Mutual diffusion coefficients for the systems water-enthanol and waterpropan-1-ol at $25^{\circ}$ C, J. Chem. Soc. Faraday Trans., vol. 89, No. 12, (1993), pp. 1969-1974.

Harris, K.R., and Newitt, P.J., Diffusion and Structure in Dilute Aqueous Alcohol Solutions: Evidence for the Effects of Large Apolar Solutes on Water, J. Phys. Chem. B, vol. 102, No. 44, (1998), pp. 8874-8879.

Herráez, J.V., and Belda, R., Refractive indices, densities and excess molar volumes of monoalcohols + water, Journal of Solution Chemistry, vol. 35, No. 9, (2006), pp. 1315-1328.

Komiya, A., and Maruyama, S., Precise and short-time measurement method of mass diffusion coefficients, Experimental Thermal and Fluid Science, vol. 30, No. 6, (2006), pp. 535-543.

Königer, A., Meier, B., and Köhler, W., Measurement of the soret, diffusion, and thermal diffusion coefficients of three binary organic benchmark mixtures and of ethanol-water mixtures using a beam deflection technique, Philosophical Magazine., vol. 89, No. 10, (2009), pp. 907-923.

Moggridge, G.D., Prediction of the mutual diffusivity in binary non-ideal liquid mixtures from the tracer diffusion coefficients, Chemical Engineering Science, vol. 71, (2012), pp. 226-238.

Okajima, J., Komiya, A., and Maruyama, S., Determination of the Mass Diffusion Coefficient by the Measurement of Concentration with Phase Shifting Interferometer and the Inverse Analysis, Proceedings of the 29th Japan Society of Thermophysical Properties, (2008), D203.

Pratt, K., and Wakeham, W., The mutual diffusion coefficient of ethano-1-water mixtures: determination by a rapid, new method, Proceedings of the Royal Society of London B., vol. 336, (1974), pp. 393-406.

Sato, K., Komiya, A., Okajima, J., and Maruyama, S., Proceedings of 9th World Conference on Experimental Heat Transfer, Fluid Mechanics and Thermodynamics(Ex-HFT 9), (2017), OC133.

SUNTORY News release 2015. [Online]. Available: https://www.suntory.co.jp/news/2015/12432.html. [Accessed: 06Jan-2018].

Torres, J.F., Komiya, A., Shoji, E., Okajima, J., and Maruyama, S., Development of phase-shifting interferometry for measurement of isothermal diffusion coefficients in binary solutions, Optics and Lasers in Engineering, vol. 50, No. 9, (2012), pp. 1287-1296.

Zhu, Q., D’Agostino, C., Ainte, M., Mantle, M.D., Gladden, L.F., Ortona, O., Paduano, L., Ciccarelli, D., and Moggridge, G.D., Prediction of mutual diffusion coefficients in binary liquid systems with one self-associating component from viscosity data and intra-diffusion coefficients at infinite dilution, Chemical Engineering Science, vol. 147, (2016), pp. 118-127. 